\title{
CHARACTERIZATION OF PSEUDO-EFFECTIVE VECTOR BUNDLES BY SINGULAR HERMITIAN METRICS
}

\author{
MASATAKA IWAI
}

\begin{abstract}
Aвstract. In this paper, we give complex geometric descriptions of the notions of algebraic geometric positivity of vector bundles and torsion-free coherent sheaves, such as nef, big, pseudoeffective, and weakly positive, by using singular Hermitian metrics. As an applications, we obtain a generalization of Mori's result. We also give a characterization of the augmented base locus by using singular Hermitian metrics on vector bundles and the Lelong numbers.
\end{abstract}

\section{CONTENTS}

1. Introduction 1

2. Definition and related results $\quad 3$

3. A singular Hermitian metric on $O_{\mathbb{P}(E)}(1) \quad 5$

4. Proof of Theorem 1.2 and Corollary $1.3 \quad 7$

5. On the case of torsion-free coherent sheaves 11

6. An augmented base locus and a Griffiths semipositive singular Hermitian metric. 12

6.1. An augmented base locus and a restiricted base locus 12

6.2. A Lelong number of a singular Hermitian metric on a vector bundle 14

6.3. A higher rank analogy of Boucksom's non-Kähler locus 15

$\begin{array}{ll}\text { References } & 17\end{array}$

\section{INTRODUCTION}

In [Kod54], Kodaira proved that a line bundle $L$ is ample if and only if $L$ has a smooth Hermitian metric with positive curvature. After that, Demailly [Dem92] gave complex geometric descriptions of nef, big, and pseudo-effective line bundles. For example, he proved that a line bundle $L$ is pseudo-effective if and only if $L$ has a singular Hermitian metric with semipositive curvature current. Ample, nef, big, and pseudo-effective are notions of algebraic geometric positivity. Thus, their works related algebraic geometry to complex geometry.

The aim of this paper is to give complex geometric descriptions of notions of algebraic geometric positivity of vector bundles and torsion-free coherent sheaves. Griffiths [Gri69] proved that if a vector bundle $E$ has a Griffiths positive metric, then $E$ is ample (i.e. $O_{\mathbb{P}(E)}(1)$ is ample). The inverse implication is unknown. We do not know whether an ample vector bundle has a Griffiths positive metric. This is so-called Griffiths' conjecture, which is one of longstanding open problems. In recent years, Liu, Sun, and Yang [LSY13] gave a partial answer to this conjecture.

2010 Mathematics Subject Classification. Primary 32J25, Secondary 14J60, 14E30.

Key words and phrases. Singular Hermitian metrics of vector bundle, Pseudo-effective, Weakly positive, Big . 
Theorem 1.1. [LSY13, Theorem 1.2 and Corollary 4.6] Let X be a smooth projective variety and $E$ be a holomorphic vector bundle on $X$. If $E$ is ample, then there exists $k \in \mathbb{N}_{>0}$ such that $\operatorname{Sym}^{k}(E)$ has a Griffiths (Nakano) positive smooth Hermitian metric.

Throughout this paper, we will denote by $\operatorname{Sym}^{k}(E)$ the $k$-th symmetric power of $E$ and denote by $\mathbb{N}_{>0}$ the set of positive integers. Inspired by the works of Liu, Sun, and Yang, we study notions of algebraic geometric positivity of vector bundles by using smooth and singular Hermitian metrics.

THEOREM 1.2. Let $X$ be a smooth projective variety and $E$ be a holomorphic vector bundle on $X$.

(1) E is nef iff there exists an ample line bundle A on $X$, such that $\operatorname{Sym}^{k}(E) \otimes A$ has a Griffiths semipositive smooth Hermitian metric for any $k \in \mathbb{N}_{>0}$.

(2) $E$ is big * iff there exist an ample line bundle $A$ and $k \in \mathbb{N}_{>0}$, such that $\operatorname{Sym}^{k}(E) \otimes A^{-1}$ has a Griffiths semipositive singular Hermitian metric.

(3) $E$ is pseudo-effective iff there exists an ample line bundle A, such that $\operatorname{Sym}^{k}(E) \otimes A$ has a Griffiths semipositive singular Hermitian metric for any $k \in \mathbb{N}_{>0}$.

(4) E is weakly positive iff there exist an ample line bundle A and a proper Zariski closed set $Z$, such that $\operatorname{Sym}^{k}(E) \otimes A$ has a Griffiths semipositive singular Hermitian metric $h_{k}$ for any $k \in \mathbb{N}_{>0}$ and the Lelong number of $h_{k}$ at $x$ is less than 2 for any $x \in X \backslash Z$.

We will explain the definitions of big, pseudo-effective, and weakly positive in Section 2. Further, we obtain similar results in the case of torsion-free coherent sheaves. We will discuss about torsion-free coherent sheaves in Section 5.

Nef, big, pseudo-effective, and weakly positive are notions of algebraic geometric positivity of vector bundles and torsion-free coherent sheaves. In particular, Viehweg [Vie83a] proved that a direct image sheaf of an $m$-th relative canonical line bundle $f_{*}\left(m K_{X / Y}\right)$ is weakly positive for any fibration $f: X \rightarrow Y$. By using this result, he studied Iitaka's conjecture. A Griffiths semipositive singular Hermitian metric, which is an analogy of a singular Hermitian metric of a line bundle and a Griffiths semipositive metric, was investigated in many papers. By using Griffiths semipositive singular Hermitian metrics, Cao and Păun [CP17] proved Iitaka's conjecture when the base space is an Abelian variety. Therefore, our results also relate algebraic geometry to complex geometry.

We have the following corollary.

Corollary 1.3. Let $X$ be a smooth projective n-dimensional variety. If the tangent bundle $T_{X}$ is big, then $X$ is biholomorphic to $\mathbb{P}^{n}$.

This corollary is a generalization of Mori's result: "If the tangent bundle $T_{X}$ is ample, then $X$ is biholomorphic to $\mathbb{P}^{n}$ ", since an ample vector bundle is big. This corollary was proved by Fulger and Murayama [FM21, Corollary 7.8] by using Seshadri constants of vector bundles . We give an another proof by using singular Hermitian metrics. Finally, we investigate the relationships between augmented base loci and Griffiths semipositive singular Hermitian metrics of vector bundles.

Acknowledgment. The author would like to thank his supervisor Prof. Shigeharu Takayama for helpful comments and enormous support. He would like to thank Prof. Takayuki Koike for useful comments. He also would like to thank anonymous referee for some suggestions about

\footnotetext{
*The definition of "big" in this paper is different from the definition of "big" in [Laz04b].
} 
the contents in Chapter 6. This work is supported by the Program for Leading Graduate Schools, MEXT, Japan. This work is also supported by JSPS KAKENHI Grant Number 17J04457.

\section{Definition AND Related Results}

Let $X$ be a smooth projective variety. A function $\varphi: X \rightarrow[-\infty,+\infty)$ is said to be quasiplurisubharmonic if $\varphi$ is locally the sum of a plurisubharmonic function and a smooth function. Let $L$ be a line bundle on $X$. Fix a smooth metric $h_{0}$ on $L . h$ is a singular Hermitian metric if $h=h_{0} e^{-\varphi}$ for some quasi-plurisubharmonic function $\varphi$.

For any quasi-plurisubharmonic function $\varphi$ on $X$, the multiplier ideal sheaf $\mathcal{J}\left(e^{-\varphi}\right)$ is a coherent subsheaf of $O_{X}$ defined by

$$
\mathcal{J}\left(e^{-\varphi}\right)_{x}:=\left\{f \in O_{X, x} ; \exists U \ni x, \int_{U}|f|^{2} e^{-\varphi} d \lambda<\infty\right\},
$$

where $U$ is an open coordinate neighborhood of $x$ and $d \lambda$ is the standard Lesbegue measure in the corresponding open chart of $\mathbb{C}^{n}$. The Lelong number $v(\varphi, x)$ at $x \in X$ is defined by

$$
v(\varphi, x):=\liminf _{z \rightarrow x} \frac{\varphi(z)}{\log |z-x|} .
$$

We define the multiplier ideal sheaf $\mathcal{J}(h)$ of a singular Hermitian metric $h$ on $L$ by $\mathcal{J}(h):=$ $\mathcal{J}\left(e^{\log \left(h h_{0}^{-1}\right)}\right)$, and define the Lelong number $v(h, x)$ of $h$ at $x \in X$ by $v(h, x):=v\left(-\log \left(h h_{0}^{-1}\right), x\right)$. We point out that both $\mathcal{J}(h)$ and $v(h, x)$ do not depend on the choice of $h_{0}$.

We review the definitions of singular Hermitian metrics of vector bundles. Let $H_{r}$ be the set of $r \times r$ semipositive definite Hermitian matrixs and $\bar{H}_{r}$ be the space of semipositive, possibly unbounded Hermitian forms on $\mathbb{C}^{r}$.

Definition 2.1. [PT18, Definition 2.2.1 and 2.2.2]

(1) A singular Hermitian metric $h$ on $E$ is defined to be a locally measurable map with values in $\bar{H}_{r}$ such that $0<\operatorname{det} h<+\infty$ almost everywhere.

(2) A singular Hermitian metric $h$ on $E$ is Griffiths seminegative (negatively curved) if the function $\log |u|_{h}^{2}$ is plurisubharmonic for any local section $u$ of $E$.

(3) A singular Hermitian metric $h$ on $E$ is Griffiths semipositive (positively curved) if the dual singular Hermitian metric $h^{*}:=h^{-1}$ on the dual vector bundle $E^{*}$ is Griffiths seminegative.

These definitions are well-defined even if $E$ is a line bundle. In particular, for any singular Hermitian metric $h$ on a line bundle $L, h$ is Griffiths semipositive iff $h$ has semipositive curvature current. We recall the definition of a singular Hermitian metric on a torsion-free coherent sheaf. Let $\mathcal{F} \neq 0$ be a torsion-free coherent sheaf on $X$. We will denote by $X_{\mathcal{F}}$ the maximal Zariski open set where $\mathcal{F}$ is locally free.

DeFinition 2.2. [PT18, Definition 2.4.1]

(1) A singular Hermitian metric $h$ on $\mathcal{F}$ is a singular Hermitian metric on the vector bundle $\left.\mathcal{F}\right|_{X_{\mathcal{F}}}$.

(2) A singular Hermitian metric $h$ on $\mathcal{F}$ is Griffiths seminegative (negatively curved) if $\left.h\right|_{X_{\mathcal{F}}}$ is Griffiths seminegative.

(3) A singular Hermitian metric $h$ on $\mathcal{F}$ is Griffiths semipositive (positively curved) if there exists a Griffiths seminegative metric $g$ on $\left.\mathcal{F}^{*}\right|_{X_{\mathcal{F}}}$ such that $\left.h\right|_{X_{\mathcal{F}}}=\left(\left.g\right|_{X_{\mathcal{F}}}\right)^{*}$ 
These are well-defined definitions (see [PT18, Remark 2.4.2]). About a Griffiths semipositive singular Hermitian metric, Păun and Takayama proved the following Theorem.

Theorem 2.3. [PT18, Theorem 1.1] [HPS18, Theorem 21.1 and Corollary 21.2] Let $f: X \rightarrow$ $Y$ be a projective surjective morphism between connected complex manifolds and $(L, h)$ be a holomorphic line bundle with a singular Hermitian metric with semipositive curvature current on $X$. Then $f_{*}\left(K_{X / Y} \otimes L \otimes \mathcal{J}(h)\right)$ has a Griffiths semipositive singular Hermitian metric.

Moreover if the inclusion morphism

$$
f_{*}\left(K_{X / Y} \otimes L \otimes \mathcal{J}(h)\right) \rightarrow f_{*}\left(K_{X / Y} \otimes L\right)
$$

is generically isomorphism, then $f_{*}\left(K_{X / Y} \otimes L\right)$ also has a Griffiths semipositive singular Hermitian metric.

Finally we recall some notions of algebraic positivity of torsion-free coherent sheaves.

Definition 2.4. [Vie83a] [Vie83b] [Nak04][BDPP13] Let $X$ be a smooth projective variety and $\mathcal{F}$ be a torsion-free coherent sheaf on $X$.

(1) $\mathcal{F}$ is weakly positive at $x \in X$ if for any $a \in \mathbb{N}_{>0}$ and any ample line bundle $A$, there exists $b \in \mathbb{N}_{>0}$ such that $\widehat{\operatorname{Sym}}^{a b}(\mathcal{F}) \otimes A^{b}$ is globally generated at $x$.

(2) $\mathcal{F}$ is pseudo-effective (weakly positive in the sense of Nakayama) if there exists $x \in X$ such that $\mathcal{F}$ is weakly positive at $x$.

(3) $\mathcal{F}$ is weakly positive (weakly positive in the sense of Viehweg) if there exists a Zariski open set $U \subset X$ such that $\mathcal{F}$ is weakly positive at any $x \in U$.

(4) $\mathcal{F}$ is big (big in the sense of Viehweg) if there exist an ample line bundle $A$ and $a \in \mathbb{N}_{>0}$ such that $\widehat{\operatorname{Sym}}^{a}(\mathcal{F}) \otimes A^{-1}$ is pseudo-effective.

We will denote by $\widehat{\operatorname{Sym}}^{k}(\mathcal{F})$ the double dual of the $k$-th symmetric power of $\mathcal{F}$. These definitions are well-defined even if $\mathcal{F}$ is a line bundle.

Lemma 2.5. Let $X$ be a smooth projective variety and $\mathcal{F}$ be a torsion-free coherent sheaf on $X$. If $\mathcal{F}$ is big, then $\mathcal{F}$ is weakly positive.

Proof. By the assumption, there exist $k \in \mathbb{N}_{>0}$ and an ample line bundle $A$ such that $\widehat{\operatorname{Sym}}^{k}(\mathcal{F}) \otimes$ $A^{-1}$ is pseudo-effective. We may assume that $A$ is very ample. Then we can take $l \in \mathbb{N}_{>0}$ and $x \in X$ such that $\widehat{\operatorname{Sym}}^{k l}(\mathcal{F}) \otimes A^{1-l}$ is globally generated at $x$. Hence there exists a Zariski open set $U \subset X$ such that $\widehat{\operatorname{Sym}}^{k l}(\mathcal{F})$ is globally generated on $U$. Therefore, for any $a \in \mathbb{N}_{>0}, \widehat{\operatorname{Sym}}^{a k l}(\mathcal{F}) \otimes A$ is globally generated on $U$.

For any vector bundle $E$, we obtain the following implication:

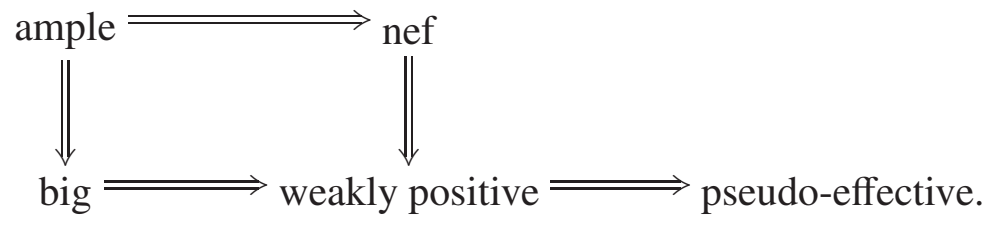




\section{A singular Hermitian Metric on $O_{\mathbb{P}(E)}(1)$}

Throughout this paper, we will denote by $\pi: \mathbb{P}(E) \rightarrow X$ the projective bundle of rank one quotients of $E$, and denote by $\mathcal{O}_{\mathbb{P}(E)}(1)$ the universal quotient of $\pi^{*} E$ on $\mathbb{P}(E)$. We study a singular Hermitian metric on $\mathcal{O}_{\mathbb{P}(E)}(1)$ induced by a singular Hermitian metric on $E$.

Lemma 3.1. Let $X$ be a smooth projective $n$-dimensional variety, $E$ be a holomorphic vector bundle of rank $r$ on $X$, and $A$ be a line bundle on $X$. Assume that there exists $m \in \mathbb{N}_{>0}$ such that $\operatorname{Sym}^{m}(E) \otimes A$ has a Griffiths semipositive singular Hermitian metric $h_{m}$. Then $O_{\mathbb{P}(E)}(m) \otimes \pi^{*} A$ has a singular Hermitian metric $g_{m}$ with semipositive curvature current.

Moreover, for any $x \in X$, there exist an open set $V$ near $x$ and a positive constant $C_{V}$, such that $g_{m} \leq C_{V} \pi^{*}\left(\operatorname{det} h_{m}\right)$ on $\pi^{-1}(V)$.

Proof. We will denote by $\pi_{m}: \mathbb{P}\left(\operatorname{Sym}^{m}(E) \otimes A\right) \rightarrow X$. Notice that $\mathbb{P}\left(\operatorname{Sym}^{m}(E)\right)=\mathbb{P}\left(\operatorname{Sym}^{m}(E) \otimes\right.$ $A)$ and $O_{\mathbb{P}\left(\operatorname{Sym}^{m}(E)\right)}(1) \otimes \pi_{m}^{*}(A)=O_{\mathbb{P}\left(\operatorname{Sym}^{m}(E) \otimes A\right)}(1)$. Let $\mu_{m}: \mathbb{P}(E) \rightarrow \mathbb{P}\left(\operatorname{Sym}^{m}(E)\right)$ be a standard $m$-th Veronese embedding. Then $\pi=\pi_{m} \circ \mu_{m}$ and

$$
O_{\mathbb{P}(E)}(m) \otimes \pi^{*} A=\mu_{m}^{*}\left(O_{\mathbb{P}\left(\operatorname{Sym}^{m}(E)\right)}(1)\right) \otimes \pi^{*}(A)=\mu_{m}^{*}\left(O_{\mathbb{P}\left(\operatorname{Sym}^{m}(E) \otimes A\right)}(1)\right) .
$$

By [PT18, Proposition 2.3.5], $\mathcal{O}_{\mathbb{P}\left(\operatorname{Sym}^{m}(E) \otimes A\right)}(1)$ admits a singular Hermitian metric $\widetilde{g_{m}}$ with semipositive curvature current. Therefore $g_{m}:=\pi_{m}^{*} \widetilde{g_{m}}$ is a singular Hermitian metric with semipositive curvature current on $O_{\mathbb{P}(E)}(m) \otimes \pi^{*} A$.

Fix $x \in X$. Since $\pi_{m}^{-1}(x)$ is compact, there exist an open set $V$ near $x$ and a positive constant $C_{V}$ such that $\widetilde{g_{m}} \leq C_{V} \pi_{m}^{*}\left(\operatorname{det} h_{m}\right)$ on $\pi_{m}^{-1}(V)$ by [PT18, Proposition 2.3.5]. Therefore, we have $g_{m} \leq C_{V} \pi^{*}\left(\operatorname{det} h_{m}\right)$ on $\pi^{-1}(V)$.

Lemma 3.2. Let $X$ be a smooth projective $n$-dimensional variety, $E$ be a holomorphic vector bundle of rank $r$ on $X$, and $A$ be a line bundle on $X$. Assume that there exist $m \in \mathbb{N}_{>0}$ and $x \in X$ such that $\operatorname{Sym}^{m}(E) \otimes A$ is globally generated at $x$. Then there exist a singular Hermitian metric $g$ with semipositive curvature current on $\mathcal{O}_{\mathbb{P}(E)}(m) \otimes \pi^{*} A$ and a proper Zariski closed set $Z \subset X$, such that $g$ is smooth outside $\pi^{-1}(Z)$.

Moreover if there exists a Zariski open set $U \subset X$ such that $\operatorname{Sym}^{m}(E) \otimes A$ is globally generated on $U$, we can take $Z$ such that $Z \cap U=\varnothing$.

Proof. Let $\left\{U_{i}\right\}$ be a finite open cover of $X$ such that $U_{i}$ is a coordinate neighborhood and $\pi^{-1}\left(U_{i}\right)$ is biholomorphic to $U_{i} \times \mathbb{P}^{r-1}$. We take a local holomorphic frame $e_{1}, \ldots, e_{r}$ of $E$ on $U_{i}$ and a local holomorphic frame $e_{A}$ of $A$ on $U_{i}$. Let $s_{1}, \ldots, s_{N}$ be a basis on $H^{0}\left(X, \operatorname{Sym}^{m}(E) \otimes A\right)$. Set $M:=\left(\begin{array}{c}m+r-1 \\ r\end{array}\right)$ and

$$
s_{j}=\sum_{\alpha} f_{j \alpha} e_{1}^{\alpha_{1}} \cdots e_{r}^{\alpha_{r}} e_{A},
$$

where $f_{j \alpha}$ are holomorphic function on $U_{i}$ and the sum is taken over $\alpha=\left(\alpha_{1}, \cdots, \alpha_{r}\right) \in \mathbb{N}_{>0}^{r}$ with $\alpha_{1}+\cdots+\alpha_{r}=m$. The $N \times M$ matrix $B^{(i)}$ is defined by $B^{(i)}=\left(f_{j \alpha}\right)$. Set $Z_{i}:=\{z \in$ $U_{i}$ : rank $\left.B^{(i)}(z)<M\right\}$ and $Z:=\cup Z_{i}$. Since $\operatorname{Sym}^{m}(E) \otimes A$ is globally generated at $x$, we have $N \geq M$ and $Z$ is a proper Zariski closed set of $X$. We define the singular Hermitian metric $g$ with semipositive curvature current on $O_{\mathbb{P}(E)}(m) \otimes \pi^{*} A$, induced by the global sections $\pi^{*}\left(s_{1}\right), \cdots, \pi^{*}\left(s_{N}\right) \in H^{0}\left(\mathbb{P}(E), O_{\mathbb{P}(E)}(m) \otimes \pi^{*} A\right)$ (see [Dem12, Example 3.14]).

We show that $g$ is smooth outside $\pi^{-1}(Z)$. We will denote by $e_{1}^{*}, \ldots, e_{r}^{*}$ the dual frame on $E^{*}$. The corresponding holomorphic coordinate on $E^{*}$ are denoted by $\left(W_{1}, \cdots, W_{r}\right)$. We may regard 
$\pi^{-1}\left(U_{i}\right)$ as $U_{i} \times \mathbb{P}^{r-1}$. We take the chart $\left\{\left[W_{1}: \cdots: W_{r}\right] \in \mathbb{P}^{r-1}: W_{r} \neq 0\right\}$. We will define the isomorphism by

$$
\begin{array}{ccc}
U_{i} \times\left\{W_{r} \neq 0\right\} & \rightarrow & U_{i} \times \mathbb{C}^{r-1} \\
\left(z,\left[W_{1}: \cdots: W_{r}\right]\right) & \rightarrow & \left(z, \frac{W_{1}}{W_{r}}, \cdots, \frac{W_{r-1}}{W_{r}}\right)
\end{array}
$$

and we regard $U_{i} \times\left\{W_{r} \neq 0\right\}$ as $U_{i} \times \mathbb{C}^{r-1}$. Put $\eta_{l}:=\frac{W_{l}}{W_{r}}$ for $1 \leq l \leq r-1$ and $\eta_{r}:=1$. In this setting, we have

$$
\left.\mathcal{O}_{\mathbb{P}(E)}(-1)\right|_{U_{i} \times \mathbb{C}^{r-1}}=\left\{(z, \eta, \xi) \in U_{i} \times \mathbb{C}^{r-1} \times \mathbb{C}^{r}: \eta_{i} \xi_{j}=\eta_{j} \xi_{i}\right\}
$$

and the local section

$$
e_{O_{\mathbb{P}(E)}(-1)}\left(z,\left(\eta_{1}, \cdots, \eta_{r-1}\right)\right)=\left(z,\left(\eta_{1}, \cdots, \eta_{r-1}\right),\left(\eta_{1}, \cdots, \eta_{r-1}, 1\right)\right) .
$$

The local section $e_{O_{\mathbb{P}(E)}(1)}$ of $\mathbb{P}(E)(1)$ is defined by the dual of $e_{O_{\mathbb{P}(E)}(-1)}$. Then we have

$$
\left.\pi^{*}\left(s_{j}\right)\right|_{U_{i} \times \mathbb{C}^{r-1}}=\sum_{\alpha} f_{j \alpha}(z) \eta_{1}^{\alpha_{1}} \cdots \eta_{r-1}^{\alpha_{r-1}} 1^{\alpha_{r}} e_{O_{\mathbb{P}(E)}(1)}^{m} \pi^{*}\left(e_{A}\right)
$$

by using the isomorphism $H^{0}\left(X, \operatorname{Sym}^{m}(E) \otimes A\right) \simeq H^{0}\left(\mathbb{P}(E), O_{\mathbb{P}(E)}(m) \otimes \pi^{*}(A)\right)$.

Since $g$ is defined by $1 /\left(\sum_{1 \leq j \leq N}\left|\pi^{*}\left(s_{j}\right)\right|^{2}\right), g$ is described on $U_{i} \times \mathbb{C}^{r-1}$ by

$$
g\left(z, \eta_{1}, \cdots, \eta_{r-1}\right)=\left(\sum_{1 \leq j \leq N}\left|\sum_{\alpha} f_{j \alpha}(z) \eta_{1}^{\alpha_{1}} \cdots \eta_{r-1}^{\alpha_{r-1}} 1^{\alpha_{r}}\right|^{2}\right)^{-1} .
$$

Therefore it is enough to show that $g^{-1}\left(z, \eta_{1}, \cdots, \eta_{r-1}\right) \neq 0$ for any $\left(z, \eta_{1}, \cdots, \eta_{r-1}\right) \in\left(U_{i} \backslash W\right) \times$ $\mathbb{C}^{r-1}$. It is easily to check by the definition of $Z$ and the standard linear algebra.

The second statement is also easily proved by the definition of $Z$.

COROllary 3.3. Let $X$ be a smooth projective $n$-dimensional variety, $E$ be a holomorphic vector bundle of rank $r$ on $X$, and $A$ be a line bundle on $X$. Assume that there exist $m, b \in \mathbb{N}_{>0}$ and $x \in X$ such that $\operatorname{Sym}^{(m+r) b}(E) \otimes\left(A \otimes \operatorname{det} E^{*}\right)^{b}$ is globally generated at $x$. Then there exist $a$ Griffiths semipositive singular Hermitian metric $h$ on $\operatorname{Sym}^{m}(E) \otimes A$ and a proper Zariski closed set $Z \subset X$ such that $h$ is smooth outside $Z$.

Moreover if there exists a Zariski open set $U \subset X$ such that $\operatorname{Sym}^{(m+r) b}(E) \otimes\left(A \otimes \operatorname{det} E^{*}\right)^{b}$ is globally generated on $U$, we can take $Z$ such that $Z \cap U=\varnothing$.

Proof. By Lemma 3.2 and dividing by $b$, there exist a singular Hermitian metric $g$ with semipositive curvature current on $O_{\mathbb{P}(E)}(m+r) \otimes \pi^{*}\left(A \otimes \operatorname{det} E^{*}\right)$ and a proper Zariski closed set $Z \subset X$ such that $g$ is smooth outside $\pi^{-1}(Z)$. From $\operatorname{det} E \simeq \pi_{*}\left(K_{\mathbb{P}(E) / X} \otimes O_{\mathbb{P}(E)}(r)\right)$, we have

$$
\operatorname{Sym}^{m}(E) \otimes A \simeq \pi_{*}\left(K_{\mathbb{P}(E) / X} \otimes O_{\mathbb{P}(E)}(m+r) \otimes \pi^{*}\left(A \otimes \operatorname{det} E^{*}\right)\right)
$$

and the inclusion morphism

$\pi_{*}\left(K_{\mathbb{P}(E) / X} \otimes O_{\mathbb{P}(E)}(m+r) \otimes \pi^{*}\left(A \otimes \operatorname{det} E^{*}\right) \otimes \mathcal{J}(g)\right) \rightarrow \pi_{*}\left(K_{\mathbb{P}(E) / X} \otimes O_{\mathbb{P}(E)}(m+r) \otimes \pi^{*}\left(A \otimes \operatorname{det} E^{*}\right)\right)$

is generically isomorphism. By Theorem 2.3, $\operatorname{Sym}^{m}(E) \otimes A$ has a Griffiths semipositive singular Hermitian metric $h$ such that $h$ is smooth outside $Z$ (see [HPS18, Chapter 22]).

The proof of the second statement is same as above. 


\section{Proof of Theorem 1.2 And Corollary 1.3}

In this section, we prove Theorem 1.2. First, we study a pseudo-effective vector bundle .

TheOREM 4.1. Let $X$ be a smooth projective $n$-dimensional variety and $E$ be a holomorphic vector bundle of rank $r$ on $X$. The followings are equivalent.

(A) E is pseudo-effective.

(B) There exists an ample line bundle A such that $\operatorname{Sym}^{k}(E) \otimes A$ has a Griffiths semipositive singular Hermitian metric $h_{k}$ for any $k \in \mathbb{N}_{>0}$. Moreover, for any $k \in \mathbb{N}_{>0}$, there exists a proper Zariski closed set $Z_{k} \subset X$ such that $h_{k}$ is smooth outside $Z_{k}$.

(C) There exists an ample line bundle A such that $\operatorname{Sym}^{k}(E) \otimes A$ has a Griffiths semipositive singular Hermitian metric $h_{k}$ for any $k \in \mathbb{N}_{>0}$.

Moreover if $E$ satisfies the condition $(C)$, then $E$ is weakly positive at any $x \in X \backslash \cup_{k \in \mathbb{N}_{>0}}\{z \in$ $\left.\left.X: v\left(\operatorname{det} h_{k}, z\right) \geq 2\right)\right\}$.

Proof. (A) $\Rightarrow(\mathrm{B})$. We take $x \in X$ such that $E$ is weakly positive at $x$, and take an ample line bundle $A$ such that $A \otimes \operatorname{det} E^{*}$ is ample. For any $a \in \mathbb{N}_{>0}$, there exists $b \in \mathbb{N}_{>0}$ such that $\operatorname{Sym}^{(a+r) b}(E) \otimes\left(A \otimes \operatorname{det} E^{*}\right)^{b}$ is globally generated at $x$. By Corollary 3.3, the proof is complete.

(B) $\Rightarrow(\mathrm{C})$. Clear.

$(\mathrm{C}) \Rightarrow(\mathrm{A})$. The proof will be divided into 3 steps.

Step 1. Fix an ample line bundle $H$. By Siu's Theorem [Dem12, Corollary 13.3], the set $\left.Z_{k}:=\left\{z \in X: v\left(\operatorname{det} h_{k}, z\right) \geq 2\right)\right\}$ is a proper Zariski closed set. Fix $x \in X \backslash \cup_{k} Z_{k}$. We take a local coordinate $\left(U ; z_{1}, \cdots, z_{n}\right)$ near $x$. Let $\varphi=\eta(n+1) \log |z-x|^{2}$, where $\eta$ is a cut-off function such that $\eta \equiv 1$ near $x$. Let $h_{H}$ be a positive smooth Hermitian metric on $H$. We take $b \in \mathbb{N}_{>0}$ such that

(1) $A^{-1} \otimes K_{X}^{-1} \otimes \operatorname{det} E^{*} \otimes H^{b}$ is ample, and

(2) $b \sqrt{-1} \Theta_{H, g_{H}}+\sqrt{-1} \partial \bar{\partial} \varphi \geq 0$ in the sense of current.

From $\operatorname{Sym}^{2 a b}(E) \otimes H^{2 b} \simeq \pi_{*}\left(O_{\mathbb{P}(E)}(2 a b) \otimes \pi^{*} H^{2 b}\right)$, it is enough to show that the restriction map

$$
H^{0}\left(\mathbb{P}(E), O_{\mathbb{P}(E)}(2 a b) \otimes \pi^{*} H^{2 b}\right) \rightarrow H^{0}\left(\pi^{-1}(x),\left.O_{\mathbb{P}(E)}(2 a b) \otimes \pi^{*} H^{2 b}\right|_{\pi^{-1}(x)}\right)
$$

is surjective for any $a \in \mathbb{N}_{>0}$.

Step 2. From $\pi^{*}(\operatorname{det} E) \simeq K_{\mathbb{P}(E) / X} \otimes O_{\mathbb{P}(E)}(r)$, we have

$$
O_{\mathbb{P}(E)}(2 a b) \otimes \pi^{*} H^{2 b} \simeq K_{\mathbb{P}(E)} \otimes\left(O_{\mathbb{P}(E)}(2 a b+r) \otimes \pi^{*} A\right) \otimes \pi^{*}\left(A^{-1} \otimes K_{X}^{-1} \otimes \operatorname{det} E^{*} \otimes H^{b}\right) \otimes \pi^{*}\left(H^{b}\right) .
$$

Since $\operatorname{Sym}^{2 a b+r}(E) \otimes A$ has a Griffiths semipositive singular Hermitian metric $h_{2 a b+r}$, by Lemma $3.1, \mathcal{O}_{\mathbb{P}(E)}(2 a b+r) \otimes \pi^{*} A$ has a singular Hermitian metric $g_{2 a b+r}$ with semipositive curvature current. By Skoda's theorem [Dem12, Lemma 5.6] and Lemma 3.1, there exist an open set $x \in V \subset X$ and a positive constant $C$ such that

(1) $g_{2 a b+r} \leq C \pi^{*}\left(\operatorname{det} h_{2 a b+r}\right)$ holds on $\pi^{-1}(V)$,

(2) $\operatorname{det} h_{2 a b+r} \in L^{1}(V)$, and

(3) $\varphi=(n+1) \log |z-x|^{2}$ holds on $V$.

Since $A^{-1} \otimes K_{X}^{-1} \otimes \operatorname{det} E^{*} \otimes H^{b}$ is ample, there exists a smooth positive metric $g_{1}$ on $A^{-1} \otimes$ $K_{X}^{-1} \otimes \operatorname{det} E^{*} \otimes H^{b}$. Set $\widetilde{L}:=\left(O_{\mathbb{P}(E)}(2 a b+r) \otimes \pi^{*} A\right) \otimes \pi^{*}\left(A^{-1} \otimes K_{X}^{-1} \otimes \operatorname{det} E^{*} \otimes H^{b}\right) \otimes \pi^{*}\left(H^{b}\right)$, $\widetilde{g}:=g_{2 a b+r} \pi^{*}\left(g_{1} g_{H}^{b}\right)$, and $\psi:=\frac{n}{n+1} \pi^{*} \varphi$. Then the followings hold.

(1) $K_{\mathbb{P}(E)} \otimes \widetilde{L} \simeq O_{\mathbb{P}(E)}(2 a b) \otimes \pi^{*} H^{2 b}$.

(2) $\widetilde{g}$ is a singular Hermitian metric with semipositive curvature current on $\widetilde{L}$.

(3) For any $\alpha \in[0,1]$, we have $\sqrt{-1} \Theta_{\widetilde{L}, \widetilde{g}}+\left(1+\frac{\alpha}{n}\right) \sqrt{-1} \partial \bar{\partial} \psi \geq 0$ in the sense of current. 
Step 3. Fix a Kähler form $\omega_{\mathbb{P}(E)}$ on $\mathbb{P}(E)$. If necessarily we take $V$ small enough, we may assume that $\pi^{-1}(V)$ is biholomorphic on $V \times \mathbb{P}^{r-1}$. Therefore, there exists $s_{V} \in H^{0}\left(\pi^{-1}(V), K_{\mathbb{P}(E)} \otimes \widetilde{L}\right)$ such that $\left.s_{V}\right|_{\pi^{-1}(x)}=s$. We take a cut-off function $\rho$ on $V$ such that $\rho=1$ near $x$ and $\inf _{\operatorname{supp}(\bar{\partial} \rho)} \varphi>$ $-\infty$. Set $\widetilde{\rho}:=\pi^{*} \rho$. We solve the global $\bar{\partial}$-equation $\bar{\partial} F=\bar{\partial}\left(\widetilde{\rho} s_{V}\right)$ with the weight $\widetilde{g} e^{-\psi}$.

First, we have

$$
\left\|\widetilde{\rho} s_{V}\right\|_{\widetilde{g}, \omega_{\mathbb{P}(E)}}^{2}=\int_{\pi^{-1}(V)}\left|\widetilde{\rho} s_{V}\right|_{\tilde{g}, \omega_{\mathbb{P}(E)}^{2}}^{2} d V_{\omega_{\mathbb{P}(E)}, \mathbb{P}(E)} \leq C_{1} \int_{\pi^{-1}(V)}\left|\pi^{*} \operatorname{det} h\right| d V_{\omega_{\mathbb{P}(E)}, \mathbb{P}(E)}<+\infty,
$$

where $C_{1}$ is a positive constant. Similarly, it is easy to check $\left.\| \bar{\partial} \widetilde{\rho} s_{V}\right) \|_{\tilde{g}, \omega_{\mathbb{P}(E)}}^{2}<+\infty$. Therefore $\bar{\partial}\left(\widetilde{\rho} s_{V}\right)$ gives rise to a cohomology class $\left.\left[\bar{\partial} \widetilde{\rho} s_{V}\right)\right]$ which is $\left[\bar{\partial}\left(\widetilde{\rho} s_{V}\right)\right]=0$ in $H^{1}\left(\mathbb{P}(E), K_{\mathbb{P}(E)} \otimes \widetilde{L} \otimes\right.$ $\mathcal{J}(\widetilde{g}))$.

Second, we have

$\left.\left\|\bar{\partial}\left(\widetilde{\rho} s_{V}\right)\right\|_{\tilde{g} e^{-\psi}, \omega_{\mathbb{P}(E)}}^{2}=\int_{\pi^{-1}(V)} \mid \bar{\partial} \widetilde{\rho} s_{V}\right)\left.\right|_{\tilde{g} e^{-\psi}, \omega_{\mathbb{P}(E)}^{2}} ^{2} d V_{\omega_{\mathbb{P}(E)}, \mathbb{P}(E)} \leq C_{2} \int_{\pi^{-1}(\operatorname{supp}(\bar{\partial} \rho))}\left|\pi^{*}(\operatorname{det} h)\right| e^{-\psi} d V_{\omega_{\mathbb{P}(E)}, \mathbb{P}(E)}<+\infty$,

where $C_{2}$ is a positive constant. Therefore $\bar{\partial}\left(\widetilde{\rho} s_{V}\right)$ is a $\bar{\partial}$-closed $(n+r-1,1)$ form with $\widetilde{L}$ value which is square integrable the weight of $\widetilde{g} e^{-\psi}$.

By the injectivity theorem in [CDM17, Theorem 1.5], the natural morphism

$$
H^{1}\left(\mathbb{P}(E), K_{\mathbb{P}(E)} \otimes \widetilde{L} \otimes \mathcal{J}\left(g e^{-\psi}\right)\right) \rightarrow H^{1}\left(\mathbb{P}(E), K_{\mathbb{P}(E)} \otimes \widetilde{L} \otimes \mathcal{J}(\widetilde{g})\right)
$$

is injective. From $\left.\left[\bar{\partial} \widetilde{\rho} s_{V}\right)\right]=0$ in $H^{1}\left(\mathbb{P}(E), K_{\mathbb{P}(E)} \otimes \widetilde{L} \otimes \mathcal{J}(\widetilde{g})\right)$, we have $\left[\bar{\partial}\left(\widetilde{\rho} s_{V}\right)\right]=0$ in $H^{1}\left(\mathbb{P}(E), K_{\mathbb{P}(E)} \otimes \widetilde{L} \otimes \mathcal{J}\left(\widetilde{g} e^{-\psi}\right)\right)$. Hence we obtain an $(n+r-1,1)$ form $F$ with $\widetilde{L}$ value which is square integrable with the weight $\widetilde{g} e^{-\psi}$ such that $\bar{\partial} F=\bar{\partial}\left(\widetilde{\rho} s_{V}\right)$.

We will show that $\left.F\right|_{\pi^{-1}(x)} \equiv 0$. To obtain a contradiction, we assume that $F(z) \neq 0$ for a point $z \in \pi^{-1}(x)$. We take an open set $x \in W \subset \subset V$, an open set $W^{\prime} \subset \mathbb{P}^{r-1}$, and a positive constant $C_{3}$ such that $W \times W^{\prime} \subset \subset \pi^{-1}(V)$ and $|F|_{g}^{2} \geq C_{3}$ on $W$. Thus we have

$$
\begin{aligned}
\|F\|_{\tilde{g} e^{-\psi}, \omega_{\mathbb{P}(E)}}^{2}=\int_{\mathbb{P}(E)}|F|_{\tilde{g}^{-\psi}, \omega_{\mathbb{P}(E)}}^{2} d V_{\omega_{\mathbb{P}(E)}, \mathbb{P}(E)} & \geq \int_{W \times W^{\prime}}|F|_{\tilde{g}^{-\psi}, \omega_{\mathbb{P}(E)}^{2}} d V_{\omega_{\mathbb{P}(E)}, \mathbb{P}(E)} \\
& \geq C_{4} \int_{W \times W^{\prime}} e^{-\psi} d V_{\omega_{\mathbb{P}(E)}, \mathbb{P}(E)} \\
& \geq C_{5} \int_{W \times W^{\prime}} e^{-n \log |z-x|^{2}} d V_{\omega_{\mathbb{P}(E)}, \mathbb{P}(E)} \\
& =+\infty
\end{aligned}
$$

where $C_{4}$ and $C_{5}$ are positive constants. This is a contradiction from $\|F\|_{\tilde{g} e^{-\psi}, \omega_{\mathbb{P}(E)}}^{2}<+\infty$.

Therefore we put $S:=\widetilde{\rho} s_{V}-F \in H^{0}\left(\mathbb{P}(E), K_{\mathbb{P}(E)} \otimes \widetilde{L}\right)$, then $\left.\left.S\right|_{\pi^{-1}(x)}=\widetilde{\rho} s_{V}-F\right)\left.\right|_{\pi^{-1}(x)}=s$, which completes the proof. Hence $E$ is weakly positive at any $x \in X \backslash \cup_{k} Z_{k}$.

By the same argument, we have the following corollary.

Corollary 4.2. Let $X$ be a smooth projective n-dimensional variety and $E$ be a holomorphic vector bundle of rank $r$ on $X$. The followings are equivalent.

(A) E is weakly positive. 
(B) There exist an ample line bundle $A$ and a proper Zariski closed set $Z \subset X$, such that $\operatorname{Sym}^{k}(E) \otimes A$ has a Griffiths semipositive singular Hermitian metric $h_{k}$ for any $k \in \mathbb{N}_{>0}$ and $h_{k}$ is smooth outside $Z$.

(C) There exist an ample line bundle $A$ and a proper Zariski closed set $Z \subset X$, such that $\operatorname{Sym}^{k}(E) \otimes A$ has a Griffiths semipositive singular Hermitian metric $h_{k}$ for any $k \in \mathbb{N}_{>0}$ and $\cup_{k}\left\{z \in X: v\left(\operatorname{det} h_{k}, z\right) \geq 2\right\} \subset Z$.

Proof. $(\mathrm{B}) \Rightarrow(\mathrm{C})$ is clear. By Theorem 4.1 , we obtain $(\mathrm{C}) \Rightarrow(\mathrm{A})$. We give a proof of $(\mathrm{A}) \Rightarrow$ (B). By the definition, there exists a Zariski open set $U$ such that $E$ is a weakly positive at any $x \in U$. We take an ample line bundle $A$ such that $A \otimes \operatorname{det} E^{*}$ is ample. Fix $a \in \mathbb{N}_{>0}$. For any $m \in \mathbb{N}_{>0}$, we define $Z_{m}$ by the Zariski closed set of points $x \in X$ such that $\operatorname{Sym}^{(a+r) m} E \otimes(A \otimes$ $\left.\operatorname{det} E^{*}\right)^{m}$ is not globally generated at $x$. Then we obtain $b \in \mathbb{N}_{>0}$ such that $Z_{b}=\cap_{m \in \mathbb{N}_{>0}} Z_{m}$. Thus, $\operatorname{Sym}^{(a+r) b} E \otimes\left(A \otimes \operatorname{det} E^{*}\right)^{b}$ is globally generated at any $x \in U$ by $Z_{b} \subset X \backslash U$. By Corollary 3.3, $\operatorname{Sym}^{a} E \otimes A$ has a Griffiths semipositive singular Hermitian metric $h$ which is smooth on $U$, the proof is complete.

The following corollary was already proved in [PT18]. We give an another proof.

Corollary 4.3. [PT18, Proposition 2.3.5] Let $X$ be a smooth projective variety and $E$ be a holomorphic vector bundle on $X$. If $E$ has a Griffiths semipositive singular Hermitian metric $h$, then $E$ is weakly positive at any $x \in\{z \in X: v(\operatorname{det} h, z)=0\}$. In particular, $E$ is pseudo-effective.

Proof. Since $E$ has a Griffiths semipositive singular Hermitian metric, $\operatorname{Sym}^{k}(E)$ also has a Griffiths semipositive singular Hermitian metric $\operatorname{Sym}^{k}(h)$ for any $k \in \mathbb{N}_{>0}$. Therefore, for any ample line bundle $A, \operatorname{Sym}^{k}(E) \otimes A$ has a Griffiths semipositive singular Hermitian metric $\operatorname{Sym}^{k}(h) h_{A}$, where $h_{A}$ is a smooth metric with positive curvature on $A$. Since we have

$$
\left.\cup_{k \in \mathbb{N}_{>0}}\left\{z \in X: v\left(\operatorname{det} \operatorname{Sym}^{k}(h) h_{A}, z\right) \geq 2\right)\right\}=\{z \in X: v(\operatorname{det} h, z)>0\},
$$

$E$ is weakly positive at any $x \in\{z \in X: v(\operatorname{det} h, z)=0\}$ by Theorem 4.1.

Next, we treat big vector bundles.

Corollary 4.4. Let $X$ be a smooth projective n-dimensional variety and $E$ be a holomorphic vector bundle of rank $r$ on $X$. The followings are equivalent.

(A) E is big.

(B) There exist $k \in \mathbb{N}_{>0}$, an ample line bundle $A$, and a proper Zariski closed set $Z \subset X$, such that $\operatorname{Sym}^{k}(E) \otimes A^{-1}$ has a Griffiths semipositive singular Hermitian metric $h$ and $h$ is smooth outside $Z$.

(C) There exist an ample line bundle $A$ and $k \in \mathbb{N}_{>0}$, such that $\operatorname{Sym}^{k}(E) \otimes A^{-1}$ has a Griffiths semipositive singular Hermitian metric.

Proof. $(\mathrm{A}) \Rightarrow(\mathrm{B})$. There exist an ample line bundle $A$ and $b \in \mathbb{N}_{>0}$, such that $\operatorname{Sym}^{b}(E) \otimes A^{-1}$ is pseudo-effective. By Theorem 4.1, there exists an ample line bundle $H$ such that $\operatorname{Sym}^{k b}(E) \otimes$ $A^{-k} \otimes H$ has a Griffiths semipositive singular Hermitian metric $h_{k}$ for any $k \in \mathbb{N}_{>0}$. Moreover, there exists a proper Zariski closed set $Z_{k}$ such that $h_{k}$ is smooth outside $Z_{k}$. Therefore we take $k \in \mathbb{N}_{>0}$ such that $A^{k} \otimes H^{-1}$ is ample, which completes the proof.

$(\mathrm{B}) \Rightarrow(\mathrm{C})$. Clear.

$(\mathrm{C}) \Rightarrow(\mathrm{A})$. For any $a \in \mathbb{N}_{>0}, \operatorname{Sym}^{a}\left(\operatorname{Sym}^{k}(E) \otimes A^{-1}\right) \otimes A$ has a Griffiths semipositive singular Hermitian metric. By Theorem 4.1, $\operatorname{Sym}^{k}(E) \otimes A^{-1}$ is pseudo-effective, which completes the proof. 
Proof of Corollary 1.3 . By Corollary 4.4, there exist $k \in \mathbb{N}_{>0}$, an ample line bundle $A$, and a proper Zariski closed set $Z \subset X$, such that $\operatorname{Sym}^{k}\left(T_{X}\right) \otimes A^{-1}$ has a Griffiths semipositive singular Hermitian metric $h$ which is smooth on $X \backslash Z$. Since $X$ is uniruled, it is enough to show that $K_{X}^{-1} \cdot C \geq n+1$ for any $x \in X \backslash Z$ and for any rational curve $C$ through $x$ by [CMSB02, Cor 0.4].

Fix $x \in X \backslash Z$ and a rational curve $C$ through $x$. First we will show that $\left.T_{X}\right|_{C}$ is ample. By [Laz04a, Theorem 6.4.15], it is enough to show that any quotient bundle of $\left.T_{X}\right|_{C}$ has positive degree. Fix a quotient bundle $G$ of $\left.T_{X}\right|_{C}$ and a smooth positive metric $h_{A}$ on $A$. Sym ${ }^{k} G$ has a quotient metric $h_{\mathrm{Sym}^{k} G}$ induced by $\left.\left(h h_{A}\right)\right|_{C}$ on $\operatorname{Sym}^{k}\left(\left.T_{X}\right|_{C}\right)$. Therefore $\operatorname{det} G$ has a singular Hermitian metric $h_{\operatorname{det} G}$ with positive curvature current by some root of $\operatorname{det} h_{\mathrm{Sym}^{k} G}$. We have

$$
\operatorname{deg} G=\int_{C} c_{1}(G)=\int_{C} c_{1}\left(\operatorname{det} G, h_{\operatorname{det} G}\right)=\int_{C} \frac{\sqrt{-1}}{2 \pi} \Theta_{\operatorname{det} G, h_{\operatorname{det} G}}>0,
$$

thus $\left.T_{X}\right|_{C}$ is ample.

Since $C$ is a rational curve, we obtain

$$
\left.T_{X}\right|_{C} \cong O_{C}\left(a_{1}\right) \oplus \cdots \oplus O_{C}\left(a_{n}\right)
$$

where $a_{i}$ is integer for any $1 \leq i \leq n, a_{1} \geq a_{2} \geq \cdots \geq a_{n}$, and $a_{1} \geq 2$. Since $\left.T_{X}\right|_{C}$ is ample, we have $a_{n} \geq 1$, therefore $K_{X}^{-1} \cdot C=a_{1}+\cdots+a_{n} \geq n+1$, which completes the proof.

Finally, we study a nef vector bundle.

Proposition 4.5 (cf. [DPS94] Theorem 1.12). Let X be a smooth projective variety and $E$ be a holomorphic vector bundle of rank $r$ on $X$. E is nef (i.e. $O_{\mathbb{P}(E)}(1)$ is nef) iff there exists an ample line bundle $A$ on $X$ such that $\operatorname{Sym}^{k}(E) \otimes A$ has a Griffiths positive smooth Hermitian metric for any $k \in \mathbb{N}_{>0}$.

Proof. $(\Rightarrow)$ We assume that $E$ is nef. We take an ample line bundle $H$ on $X$ such that $H \otimes \operatorname{det} E^{*}$ is ample, and take $N \in \mathbb{N}_{>0}$ such that $E \otimes\left(H \otimes \operatorname{det} E^{*}\right)^{N}$ is ample. For any $k \in \mathbb{N}_{>0}$, we have

$$
\operatorname{Sym}^{k}(E) \otimes H \otimes\left(H \otimes \operatorname{det} E^{*}\right)^{N-1} \simeq \pi_{*}\left(K_{\mathbb{P}(E) / X} \otimes \mathcal{O}_{\mathbb{P}(E)}(k+r) \otimes \pi^{*}\left(H \otimes \operatorname{det} E^{*}\right)^{N}\right) .
$$

Since $O_{\mathbb{P}(E)}(1)$ is nef, $O_{\mathbb{P}(E)}(k+r) \otimes \pi^{*}\left(H \otimes \operatorname{det} E^{*}\right)^{N}$ is ample. Therefore, $\operatorname{Sym}^{k}(E) \otimes H \otimes(H \otimes$ $\left.\operatorname{det} E^{*}\right)^{N-1}$ has a Griffiths semipositive smooth Hermitian metric for any $k \in \mathbb{N}_{>0}$. We put $A:=$ $H^{2} \otimes\left(H \otimes \operatorname{det} E^{*}\right)^{N-1}$, the proof is complete.

$(\Leftarrow)$ Let $\mu_{k}: \mathbb{P}(E) \rightarrow \mathbb{P}\left(\operatorname{Sym}^{k}(E)\right)=\mathbb{P}\left(\operatorname{Sym}^{k}(E) \otimes A\right)$ be a standard $k$-th Veronese embedding. Since $O_{\mathbb{P}\left(\operatorname{Sym}^{k}(E) \otimes A\right)}(1)$ is ample and $O_{\mathbb{P}(E)}(k) \otimes \pi^{*} A=\mu_{k}^{*}\left(O_{\mathbb{P}\left(\operatorname{Sym}^{k}(E) \otimes A\right)}(1)\right), O_{\mathbb{P}(E)}(k) \otimes \pi^{*} A$ is ample for any $k \in \mathbb{N}_{>0}$. Therefore $O_{\mathbb{P}(E)}(1)$ is nef.

EXAMPLE 4.6 (Cutkosky's criterion). Let $X$ be a smooth projective variety and $L_{1}, \ldots, L_{r}$ be holomorphic line bundles on $X$. The vector bundle $E$ is defined by $E:=\oplus_{i=1}^{r} L_{i}$. By [Laz04a, Chapter 2.3.B], we have the following criterions.

(1) $E$ is ample iff any $L_{i}$ is ample.

(2) $E$ is nef iff any $L_{i}$ is nef.

We give a generalization of Cutkosky's criterion of big and pseudo-effective.

LeMma 4.7. (1) E is big iff any $L_{i}$ is big.

(2) E is pseudo-effective iff any $L_{i}$ is pseudo-effective. Moreover E is pseudo-effective iff E has a Griffiths semipositive singular Hermitian metric. 
Proof. (1) $\left(\Rightarrow\right.$ ) If $E$ is big, then there exist an ample line bundle $A, c \in \mathbb{N}_{>0}$, and a Zariski open set $U$, such that $\operatorname{Sym}^{c}(E) \otimes A^{-1}$ is globally generated on $U$. Thus $L_{i}^{c} \otimes A^{-1}$ is globally generated on $U$ for any $1 \leq i \leq r$. Therefore $L_{i}$ is big.

$(1)(\Leftarrow)$ Let $A$ be an ample line bundle and $h_{A}$ be a smooth metric with positive curvature on $A$, such that $\omega=\sqrt{-1} \Theta_{A, h_{A}}$ is a Kähler form on $X$. Since $L_{i}$ is big, there exist a singular Hermitian metric $h_{i}$ and positive number $\epsilon_{i}$ such that $\sqrt{-1} \Theta_{L_{i}, h_{i}} \geq \epsilon_{i} \omega$. We define a singular Hermitian metric $h$ on $E$ by $h=\oplus_{i=1}^{r} h_{i}$. We take $c \in \mathbb{N}_{>0}$ such that $\min _{1 \leq i \leq r} \epsilon_{i}>2 / c$. Then $\operatorname{Sym}^{c}(E) \otimes A^{-1}$ has a Griffiths semipositive singular Hermitian metric $\operatorname{Sym}^{c}(h) h_{A}^{-1}$, which completes the proof.

(2) $(\Rightarrow)$ Fix $x \in X$ such that $E$ is weakly positive at $x$. For any ample line bundle $A$ and $a \in \mathbb{N}_{>0}$, there exists $b \in \mathbb{N}_{>0}$ such that $\operatorname{Sym}^{a b}(E) \otimes A^{b}$ is globally generated at $x$. Hence $L_{i}^{a b} \otimes A^{b}$ is globally generated at $x$ for any $1 \leq i \leq r$. Therefore $L_{i}$ is pseudo-effective.

(2) $(\Leftarrow)$ Since $L_{i}$ is pseudo-effective, $L_{i}$ has a singular Hermitian metric $h_{i}$ with semipositive curvature current. Hence $h:=\oplus_{i=1}^{r} h_{i}$ is a Griffiths semipositive singular Hermitian metric on $E$. Therefore $E$ is pseudo-effective by Corollary 4.3 .

REMARK 4.8. In general, a pseudo-effective vector bundle does not necessarily have a Griffiths semipositive singular Hermitian metric. By Hosono [Hos17, Example 5.4], there exists a nef vector bundle which does not have a Griffiths semipositive singular Hermitian metric.

Let $C$ be an elliptic curve. $E$ is defined by the nontrivial exact sequence of vector bundles:

$$
0 \rightarrow O_{C} \rightarrow E \rightarrow O_{C} \rightarrow 0 .
$$

By [HIM19, Theorem 1.4], if $E$ has a Griffiths semipositive singular Hermitian metric, then the above exact sequence splits, which is impossible.

\section{ON THE CASE OF TORSION-FREE COHERENT SHEAVES}

THEOREM 5.1. Let $X$ be a smooth projective variety and $\mathcal{F} \neq 0$ be a torsion-free coherent sheaf on $X$.

(1) $\mathcal{F}$ is pseudo-effective iff there exists an ample line bundle $A$, such that $\widehat{\operatorname{Sym}}^{k}(\mathcal{F}) \otimes A$ has a Griffiths semipositive singular Hermitian metric for any $k \in \mathbb{N}_{>0}$.

(2) $\mathcal{F}$ is weakly positive iff there exist an ample line bundle A and a Zariski open set $U \subset X$, such that $\widehat{\operatorname{Sym}}^{k}(\mathcal{F}) \otimes A$ has a Griffiths semipositive singular Hermitian metric $h_{k}$ and the Lelong number of $h_{k}$ at $x$ is less than 2 for any $x \in U$ and any $k \in \mathbb{N}_{>0}$.

(3) $\mathcal{F}$ is big iff there exist an ample line bundle $A$ and $k \in \mathbb{N}_{>0}$, such that $\widehat{\operatorname{Sym}}^{k}(\mathcal{F}) \otimes A^{-1}$ has a Griffiths semipositive singular Hermitian metric.

Proof. We put $E:=\left.\mathcal{F}\right|_{X_{\mathcal{F}}}$, which is a vector bundle on $X_{\mathcal{F}}$. Since $\widehat{\operatorname{Sym}}^{k}(\mathcal{F}) \otimes A$ is reflexive for any $k \in \mathbb{N}_{>0}$, we have

$$
H^{0}\left(X_{\mathcal{F}}, \operatorname{Sym}^{k}(E) \otimes A\right) \simeq H^{0}\left(X, \widehat{\operatorname{Sym}}^{k}(\mathcal{F}) \otimes A\right) .
$$

$(1)(\Rightarrow)$. We assume that $\mathcal{F}$ is pseudo-effective. We take $x \in X_{\mathcal{F}}$ such that $\mathcal{F}$ is weakly positive at $x$, and take an ample line bundle $A$ such that $A \otimes(\widehat{\operatorname{det} \mathcal{F}})^{*}$ is ample. For any $k \in$ $\mathbb{N}_{>0}$, there exists $b \in \mathbb{N}_{>0}$ such that $\widehat{\operatorname{Sym}}^{k b}(\mathcal{F}) \otimes\left(A \otimes(\widehat{\operatorname{det} \mathcal{F}})^{*}\right)^{b}$ is globally generated at $x$. Therefore by Equation 5.1, $\operatorname{Sym}^{k b}(E) \otimes\left(A \otimes \operatorname{det} E^{*}\right)^{b}$ on $X_{\mathcal{F}}$ is globally generated at $x$. By the argument of Corollary 3.3, there exists a Griffiths semipositive singular Hermitian metric $h$ on $\left.\left(\widehat{\operatorname{Sym}}^{k}(\mathcal{F}) \otimes A\right)\right|_{X_{\mathcal{F}}}=\operatorname{Sym}^{k}(E) \otimes A(h$ is smooth outside a countable union of proper Zariski closed 
sets). From $\operatorname{codim}\left(X \backslash X_{\mathcal{F}}\right) \geq 2, h$ extends to $X_{\left(\widehat{\operatorname{Sym}}^{k}(\mathcal{F}) \otimes A\right)}$. Therefore $\widehat{\operatorname{Sym}}^{k}(\mathcal{F}) \otimes A$ has a Griffiths semipositive singular Hermitian metric $h$.

$(1)(\Leftarrow)$. From $X_{\mathcal{F}} \subset X_{{\widehat{\left.\left(\operatorname{Sym}^{(\mathcal{F}}\right) \otimes A\right)}}^{k}}$ for any $k \in \mathbb{N}_{>0}, \operatorname{Sym}^{k}(E) \otimes A$ has a Griffiths semipositive singular Hermitian metric for any $k \in \mathbb{N}_{>0}$. By using the argument of the proof of (C) $\Rightarrow(\mathrm{A})$ in Theorem 4.1, there exists $x \in X_{\mathcal{F}}$ such that $E$ is weakly positive at $x$. (We use Demailly's $L^{2}$ estimate on a complete Kähler manifold in [Dem82, Theorem 5.1] instead of the injectivity theorem in [CDM17] since $X_{\mathcal{F}}$ may not be a weakly 1-complete. See also [PT18, Theorem 2.5.3].) Hence $\mathcal{F}$ is pseudo-effective.

(2) The proof is similar to the proof of (1) and Theorem 4.2. (We can take $h_{k}$ such that $h_{k}$ is smooth on $U \cap X_{\mathcal{F}}$ if $\mathcal{F}$ is weakly positive on $U$.)

$(3)(\Rightarrow)$. The proof is similar to the proof of (1).

$(3)(\Leftarrow)$. By Corollary 4.3 and $(1), \widehat{\operatorname{Sym}}^{2 k}(\mathcal{F}) \otimes A^{-1}$ is pseudo-effective.

We give an application of Theorem 5.1. If a torsion-free coherent sheaf $\mathcal{F}$ has a Griffiths semipositive singular Hermitian metric $h$, then $\mathcal{F}$ is pseudo-effective. Moreover if there exists a Zariski open set $U$ such that $h$ is continuous on $U \cap X_{\mathcal{F}}$, then $\mathcal{F}$ is weakly positive at any $x \in U \cap X_{\mathcal{F}}$.

Let $f: X \rightarrow Y$ be a surjective morphism between smooth projective varieties. Then for any $m \in \mathbb{N}_{>0}, f_{*}\left(m K_{X / Y}\right)$ has a Griffiths semipositive singular Hermitian metric, which is continuous over the regular locus of $f$ by [PT18, Theorem 1.1] or [HPS18, Theorem 27.1]. Therefore, $f_{*}\left(m K_{X / Y}\right)$ is weakly positive at any point of the regular locus of $f$. This was already proved in [PT18, Theorem 5.1.2].

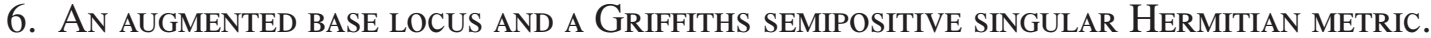

In this chapter, we study a relationship between an augmented base locus and a Griffiths semipositive singular Hermitian metric of a vector bundle.

\subsection{An augmented base locus and a restiricted base locus.}

We review some of the standard facts of augmented base loci and restiricted base loci of vector bundles.

Definition 6.1. [BKK +15 , Section 2] Let $X$ be a smooth projective variety and $E$ be a holomorphic vector bundle on $X$. The base locus of $E$ is defined by

$$
\operatorname{Bs}(E):=\left\{x \in X: H^{0}(X, E) \rightarrow E_{x} \text { is not surjective }\right\},
$$

and the stable base locus of $E$ is defined by

$$
\mathbb{B}(E):=\bigcap_{m>0} \operatorname{Bs}\left(\operatorname{Sym}^{m}(E)\right) .
$$

Let $A$ be an ample line bundle. We define the augmented base locus of $E$ by

$$
\mathbb{B}_{+}(E)=\bigcap_{m \in \mathbb{N}_{>0}} \mathbb{B}\left(\operatorname{Sym}^{m}(E) \otimes A^{-1}\right)
$$

and the restricted base locus of $E$ by

$$
\mathbb{B}_{-}(E)=\bigcup_{m \in \mathbb{N}_{>0}} \mathbb{B}\left(\operatorname{Sym}^{m}(E) \otimes A\right) .
$$


We point out that both $\mathbb{B}_{+}(E)$ and $\mathbb{B}_{-}(E)$ do not depend on the choice of the ample line bundle $A$ by $[\mathrm{BKK}+15$, Remark 2.7].

Theorem 6.2. [BKK+15, Proposition 3.1, 3.2, 7.2 and Theorem 6.4] Let X be a smooth projective variety and $E$ be a holomorphic vector bundle on $X$. Then the followings hold.

(1) $\pi\left(\mathbb{B}_{+}\left(O_{\mathbb{P}(E)}(1)\right)\right)=\mathbb{B}_{+}(E)$ and $\pi\left(\mathbb{B}_{-}\left(O_{\mathbb{P}(E)}(1)\right)\right)=\mathbb{B}_{-}(E)$.

(2) $\mathbb{B}_{+}(E) \neq X$ iff $E$ is big.

(3) $\mathbb{B}_{-}(E) \neq X$ iff $E$ is pseudo-effective.

(4) $\overline{\mathbb{B}_{-}(E)} \neq X$ iff $E$ is weakly positive, where $\overline{\mathbb{B}_{-}(E)}$ is a Zariski closure of $\mathbb{B}_{-}(E)$ in $X$.

Let $X$ be a smooth projective variety, $L$ be a line bundle, and $h$ be a singular Hermitian metric with semipositive curvature current on $L$. Let $P_{h}$ denote the set of $x \in X$ such that $v(h, x)>0$. By [Bou02, Théorème 2.1.20 and Corollaire 2.2.8], if $L$ is big, then $\mathbb{B}_{+}(L)=P_{h_{\min }}$, where $h_{\text {min }}$ is a minimal singular Hermitian metric of $L$ (see [DPS01, Theorem 1.5]).

Corollary 6.3. Let $X$ be a smooth projective variety, $E$ be a holomorphic vector bundle on $X$, $\omega$ be a Kähler form on $\mathbb{P}(E)$. Then the followings hold.

(1) $E$ is big iff $O_{\mathbb{P}(E)}(1)$ admits a singular Hermitian metric $h$ such that $\pi\left(P_{h}\right) \neq X$ and $\sqrt{-1} \Theta_{O_{\mathbb{P}(E)}(1), h} \geq \epsilon \omega$, for some $\epsilon>0$.

(2) E is pseudo-effective iff there exists a singular Hermitian metric $h_{k}$ on $O_{\mathbb{P}(E)}(1)$ such that $\pi\left(P_{h_{k}}\right) \neq X$ and $\sqrt{-1} \Theta_{O_{\mathbb{P}(E)}(1), h_{k}} \geq \frac{-\omega}{k}$ for any $k \in \mathbb{N}_{>0}$,

(3) $E$ is weakly positive iff there exists a proper Zariski closed set $Z \subset X$ such that $O_{\mathbb{P}(E)}(1)$ has a singular Hermitian metric $h_{k}$ with $\pi\left(P_{h_{k}}\right) \subset Z$ and $\sqrt{-1} \Theta_{O_{\mathbb{P}(E)}(1), h_{k}} \geq \frac{-\omega}{k}$ for any $k \in \mathbb{N}_{>0}$,

Proof. We may assume that $A$ is an ample line bundle on $\mathbb{P}(E)$ and $h_{A}$ is a smooth positive metric on $A$ such that $\sqrt{-1} \Theta_{A, h_{A}}=\omega$.

$(1)(\Rightarrow)$. By $\left[\mathrm{BKK}+15\right.$, Remark 2.7], there exists $m \in \mathbb{N}_{>0}$ such that $\mathbb{B}_{+}(E)=\pi\left(\mathbb{B}\left(O_{\mathbb{P}(E)}(m) \otimes\right.\right.$ $\left.A^{-1}\right)$. Hence $\mathcal{O}_{\mathbb{P}(E)}(m) \otimes A^{-1}$ has a singular Hermitian metric $h_{m}$ with semipositive curvature current with $\pi\left(P_{h_{m}}\right)=\mathbb{B}_{+}(E)$. Set $h:=\left(h_{m} h_{A}\right)^{\frac{1}{m}}$, which completes the proof.

$(1)(\Leftarrow)$. By the assumption, $O_{\mathbb{P}(E)}(1)$ is big. By Theorem 6.2 and [Bou02],

$$
\mathbb{B}_{+}(E)=\pi\left(\mathbb{B}_{+}\left(O_{\mathbb{P}(E)}(1)\right)\right)=\pi\left(P_{h_{\text {min }}}\right) \subset \pi\left(P_{h}\right),
$$

where $h_{\min }$ is a minimal singular Hermitian metric on $O_{\mathbb{P}(E)}(1)$. Therefore $E$ is big.

$(2)(\Rightarrow)$. Fix $k \in \mathbb{N}_{>0}$. By Theorem 6.2,

$$
\pi\left(\mathbb{B}_{+}\left(O_{\mathbb{P}(E)}(k) \otimes A\right)\right) \subset \cup_{m \in \mathbb{N}_{>0}} \pi\left(\mathbb{B}_{+}\left(O_{\mathbb{P}(E)}(m) \otimes A\right)\right)=\mathbb{B}_{-}(E) .
$$

Let $h_{\min , k}$ be a minimal singular metric on $O_{\mathbb{P}(E)}(k) \otimes A$. Set $h_{k}:=\left(h_{\min , k} h_{A}^{-1}\right)^{\frac{1}{k}}$. Then $\pi\left(P_{h_{k}}\right) \neq X$ and $\sqrt{-1} \Theta_{O_{\mathbb{P}(E)}(1), h_{k}} \geq \frac{-\omega}{k}$.

$(2)(\Leftarrow)$. Set $H_{k}:=h_{k}^{k} h_{A}$ for any $k \in \mathbb{N}_{>0}$. Then $H_{k}$ is a singular Hermitian metric with semipositve curvature current on $O_{\mathbb{P}(E)}(k) \otimes A$. By Theorem 6.2 and [Bou02],

$$
\left.\mathbb{B}_{-}(E)=\cup_{m \in \mathbb{N}_{>0}} \pi\left(\mathbb{B}_{+}\left(O_{\mathbb{P}(E)}(m) \otimes A\right)\right)\right) \subset \cup_{m \in \mathbb{N}_{>0}} \pi\left(P_{H_{m}}\right) .
$$

Therefore $E$ is pseudo-effective.

(3). The proof is same as that of (2). 
REMARK 6.4. In the hypotheses of Corollary 6.3 , if $O_{\mathbb{P}(E)}(1)$ has a singular Hermitian metric $h$ with semipositive curvature current such that $\pi\left(P_{h}\right) \neq X$, then $E$ is pseudo-effective by $\pi\left(\mathbb{B}_{-}\left(O_{\mathbb{P}(E)}(1)\right)\right) \subset \pi\left(P_{h}\right)$. However, the converse does not necessarily hold.

Let $X$ be an elliptic curve. $E$ is defined by the nontrivial exact sequence of vector bundles:

$$
0 \rightarrow O_{X} \rightarrow E \rightarrow O_{X} \rightarrow 0 .
$$

Then $E$ is nef. Let $h_{\text {min }}$ be the minimal singular Hermitian metric on $O_{\mathbb{P}(E)}(1)$. By [DPS94, Example 1.7], $h_{\min }$ has a pole and $\pi\left(P_{h_{\min }}\right)=X$.

\subsection{A Lelong number of a singular Hermitian metric on a vector bundle.}

The Lelong number of a singular Hermitian metric on a vector bundle was defined by Berndtsson [Ber20]. Based on the Berndtsson's work, we define a new Lelong number.

Let $U$ be a unit ball in $\mathbb{C}^{n}$, and $E=U \times \mathbb{C}^{r}, h$ be a Griffiths semipositive singular Hermitian metric. We take a standard frame $e_{1}, \cdots, e_{r}$ of $E$. Then we have $\mathbb{P}(E)=U \times \mathbb{P}^{r-1}$ and $O_{\mathbb{P}(E)}(1)$ can be endowed with a singular Hermitian metric $g$ with semipositive curvature current induced by $h$. We have $g=e^{-\varphi(z, W)}$, where $\varphi(z, W)$ is a quasi-plurisubharmonic function on $U \times \mathbb{P}^{r-1}$.

Definition 6.5. We will denote by $v(\varphi,(0, W))$ the Lelong number $\varphi$ at $(0, W) \in \mathbb{P}(E)$. We define the following numbers:

$$
v_{\text {sup }}(h, 0):=\sup _{W \in \mathbb{P}^{r-1}} v(\varphi,(0, W)), \quad v_{\text {inf }}(h, 0):=\inf _{W \in \mathbb{P}^{r-1}} v(\varphi,(0, W)) .
$$

We explain more explicitly. Let $h^{*}=\left(h_{i j}^{*}\right)$ be the dual metric of $h$ on $E$. We take the chart $\left\{\left[W_{1}: \cdots: W_{r}\right] \in \mathbb{P}^{r-1}: W_{r} \neq 0\right\}$ of $\mathbb{P}(E)$. As in Lemma 3.2, we define the isomorphism by

$$
\begin{array}{ccc}
U \times\left\{W_{r} \neq 0\right\} & \rightarrow & U \times \mathbb{C}^{r-1} \\
\left(z,\left[W_{1}: \cdots: W_{r}\right]\right) & \rightarrow & \left(z, \frac{W_{1}}{W_{r}}, \cdots, \frac{W_{r-1}}{W_{r}}\right) .
\end{array}
$$

We may regard $U \times\left\{W_{r} \neq 0\right\}$ as $U \times \mathbb{C}^{r-1}$. Put $\eta_{l}:=\frac{W_{l}}{W_{r}}$ for $1 \leq l \leq r-1$ and $\eta_{r}:=1$. In this setting, we have

$$
\left.\mathcal{O}_{\mathbb{P}(E)}(-1)\right|_{U \times \mathbb{C}^{r-1}}=\left\{(z, \eta, \xi) \in U \times \mathbb{C}^{r-1} \times \mathbb{C}^{r}: \eta_{i} \xi_{j}=\eta_{j} \xi_{i}\right\}
$$

and the local section

$$
e_{O_{\mathbb{P}(E)}(-1)}\left(z,\left(\eta_{1}, \cdots, \eta_{r-1}\right)\right)=\left(z,\left(\eta_{1}, \cdots, \eta_{r-1}\right),\left(\eta_{1}, \cdots, \eta_{r-1}, 1\right)\right) .
$$

Then the dual metric $g^{*}=e^{\varphi}$ on $O_{\mathbb{P}(E)}(-1)$ is written by

$$
g^{*}(z, \eta)=\left|\left(\eta_{1}, \cdots \eta_{r-1}, 1\right)\right|_{h^{*}}^{2}=\sum_{1 \leq i, j \leq r} h_{i j}^{*}(z) \eta_{i} \bar{\eta}_{j}=\frac{\sum_{1 \leq i, j \leq r} h_{i j}^{*}(z) W_{i} \bar{W}_{j}}{\left|W_{r}\right|^{2}} .
$$

Therefore, for any $W=\left[W_{1}: \cdots: W_{r}\right] \in\left\{W_{r} \neq 0\right\}$, we have

$$
\varphi(z, W)=\log \left(\sum_{1 \leq i, j \leq r} h_{i j}^{*}(z) W_{i} \bar{W}_{j}\right)-2 \log \left|W_{r}\right|
$$

and

$$
v(\varphi,(0, W))=v\left(\log \left(\sum_{1 \leq i, j \leq r} h_{i j}^{*}(z) W_{i} \bar{W}_{j}\right),(0, W)\right) .
$$


In this setting, we explain the relationship with the Lelong number defined by Berndtsson [Ber20]. For any $a=\left(a_{1}, \cdots, a_{r}\right) \in \mathbb{C}^{r} \backslash\{0\}$, we write $u_{a}=\sum_{1 \leq i \leq r} a_{i} e_{i}^{*}$. In [Ber20], the Lelong number of $h^{*}$ at 0 in the direction $u_{a}$ is defined by

$$
\gamma_{h^{*}}\left(u_{a}, 0\right)=\liminf _{z \rightarrow 0} \frac{\log \left|u_{a}\right|_{h^{*}}^{2}}{\log |z|} .
$$

From $\log \left|u_{a}\right|_{h^{*}}^{2}=\log \left(\sum_{1 \leq i, j \leq r} h_{i j}^{*}(z) a_{i} \bar{a}_{j}\right)$, we have the following corollary.

COROLlary 6.6. In the above setting, the following hold.

(1) $\gamma_{h^{*}}\left(u_{a}, 0\right)=v\left(\left.\varphi\right|_{U \times\left\{\left[a_{1}: \cdots: a_{r}\right]\right\}}, 0\right) \geq v\left(\varphi,\left(0,\left[a_{1}: \cdots: a_{r}\right]\right)\right)$ for any $a=\left(a_{1}, \cdots, a_{r}\right) \in \mathbb{C}^{r} \backslash\{0\}$.

(2) $\gamma_{h^{*}}\left(u_{a}, 0\right)=v\left(\varphi,\left(0,\left[a_{1}: \cdots: a_{r}\right]\right)\right)$ for general $a=\left(a_{1}, \cdots, a_{r}\right) \in \mathbb{C}^{r} \backslash\{0\}$.

(3) $v_{\text {inf }}(h, 0)=\inf _{a \in \mathbb{C}^{r} \backslash\{0\}} \gamma_{h^{*}}\left(u_{a}, 0\right)$.

Proof. The first equality of (1) is clear. By [Dembook, Theorem 7.13] we have the second inequality of (1) and the equality of (2).

We now prove (3). By (1), we have $v_{\text {inf }}(h, 0) \leq \inf _{a \in \mathbb{C}^{r} \backslash\{0\}} \gamma_{h^{*}}\left(u_{a}, 0\right)$. By [Dem12, Lemma 2.17], we have

$$
v_{\text {inf }}(h, 0)=v\left(\varphi,\{0\} \times \mathbb{P}^{r-1}\right)=v\left(\varphi,\left(0,\left[a_{1}: \cdots: a_{r}\right]\right)\right)
$$

for general $\left(a_{1}, \cdots, a_{r}\right) \in \mathbb{C}^{r} \backslash\{0\}$. Therefore by using (2), the proof is complete.

\subsection{A higher rank analogy of Boucksom's non-Kähler locus.}

We introduce the non-Kähler loci on vector bundles. $X$.

Definition 6.7. Let $X$ be a smooth projective variety and $E$ be a holomorphic vector bundle on

(1) For any $k \in \mathbb{N}_{>0}$ and any ample line bundle $A$, we set

$\mathcal{H}_{k, A}^{+}=\left\{h: h\right.$ is a Griffiths semipositive singular Hermitian metric of $\left.\operatorname{Sym}^{k}(E) \otimes A^{-1}\right\}$

(2) If $E$ is big, the non-Kähler locus $L_{n K}(E)$ is defined by

$$
L_{n K}(E):=\bigcap_{k, A} \bigcap_{h \in \mathcal{H}_{k, A}^{+}}\left\{x \in X: v_{\text {sup }}(h, x)>0\right\},
$$

where the cap is taken over all $k \in \mathbb{N}_{>0}$ and all ample line bundle $A$ on $X$. By Theorem 1.2 , this locus is well-defined.

This is a higher rank analogy of Boucksom's non-Kähler locus in [Bou04]. In this section, we prove the following theorem.

THEOREM 6.8. If $E$ is big, then $L_{n K}(E)=\mathbb{B}_{+}(E)$.

Therefore, we give a characterization of the augmented base loci by using singular Hermitian metrics on vector bundles and the Lelong numbers as in [Bou02, Corollaire 2.2.8].

Before the proof, we recall a singular Hermitian metric induced by holomorphic sections, proposed by Hosono [Hos 17, Chapter 4]. We assume that $E$ is a globally generated at a general point. Let $s_{1}, \ldots, s_{N} \in H^{0}(X, E)$ be holomorphic sections. We take a local coordinate $U$ and take a local holomorphic frame $e_{1}, \ldots, e_{r}$ of $E$ on $U$. Write $s_{\alpha}=\sum_{1 \leq j \leq r} f_{\alpha j} e_{j}$, where $f_{\alpha j}$ are holomorphic functions on $U$. A singular Hermitian metric $h_{s}$ induced by $s_{1}, \ldots, s_{N}$ is given by

$$
\left(h_{s}^{*}\right)_{j k}:=\sum_{1 \leq \alpha \leq N} f_{\alpha j} \overline{f_{\alpha k}} \cdot
$$

By [Hos17, Example 3.6 and Proposition 4.1], $h_{s}$ is Griffiths semipositive. 
Proposition 6.9. In this setting, $\left\{x \in X: v_{\text {sup }}\left(h_{s}, x\right)>0\right\} \subset B s(E)$.

Proof. The $N \times r$ matrix $A$ is defined by $A_{\alpha j}=f_{\alpha j}$ as in Lemma 3.2. By the standard linear algebra, we have $B s(E) \cap U=\{x \in U$ : rank $A(x)<r\}$.

Let $g=e^{-\varphi}$ be a singular Hermitian metric with semipositive curvature current on $O_{\mathbb{P}(E)}(1)$ induced by $h_{s}$. By the above argument, we have

$$
v(\varphi,(z, W))=v\left(\log \left(\sum_{1 \leq j, k \leq r, 1 \leq \alpha \leq N} f_{\alpha j} W_{j} \overline{f_{\alpha k} W_{k}}\right),(0, W)\right) .
$$

If $v_{\text {sup }}\left(h_{s}, x\right)>0$, there exists $a \in \mathbb{P}^{r-1}$ such that $v(\varphi,(x, a))>0$. We obtain

$$
\sum_{1 \leq j, k \leq r, 1 \leq \alpha \leq N} f_{\alpha j}(x) a_{j} \overline{f_{\alpha k}(x) a_{k}}=0
$$

and consequently $\sum_{1 \leq j \leq r} f_{\alpha j}(x) a_{j}=0$ for any $1 \leq \alpha \leq N$. Hence rank $A(x)<r$, therefore $x \in B s(E)$.

Now, we prove the Theorem 6.8.

Proof. First we show that $L_{n K}(E) \subset \mathbb{B}_{+}(E)$. We take a sufficiently ample line bundle $A$ such that $\mathbb{B}_{+}(E)=\bigcap_{q \in \mathbb{N}_{>0}} \mathbb{B}\left(\operatorname{Sym}^{q}(E) \otimes A^{-1}\right)$ by $[\mathrm{BKK}+15$, Remark 2.7]. It is enough to show that $L_{n K}(E) \subset \mathbb{B}\left(\operatorname{Sym}^{q}(E) \otimes A^{-1}\right)$ for any $q \in \mathbb{N}_{>0}$ such that $\mathbb{B}\left(\operatorname{Sym}^{q}(E) \otimes A^{-1}\right) \neq X$. Fix $q \in \mathbb{N}_{>0}$. We take $m \in \mathbb{N}_{>0}$ such that $\mathbb{B}\left(\operatorname{Sym}^{q}(E) \otimes A^{-1}\right)=B s\left(\operatorname{Sym}^{q m}(E) \otimes A^{-m}\right)$. From $B s\left(\operatorname{Sym}^{q m}(E) \otimes A^{-m}\right) \neq$ $X, \operatorname{Sym}^{q m}(E) \otimes A^{-m}$ can be endowed with a Griffiths semipositive singular Hermitian metric $h$ induced by global sections and $\left\{x \in X: v_{\text {sup }}(h, x)>0\right\} \subset B s\left(\operatorname{Sym}^{q m}(E) \otimes A^{-m}\right)$ holds. By $h \in \mathcal{H}_{q m, A^{m}}^{+}$and the definition of $L_{n K}(E)$, the proof is complete.

For inverse inclusion, we take $x \notin L_{n K}(E)$. There exist $k \in \mathbb{N}_{>0}$, an ample line bundle $A$, and a Griffiths semipositive singular Hermitian metric $h$ on $\operatorname{Sym}^{k}(E) \otimes A^{-1}$ such that $v_{\text {sup }}(h, x)=0$. We will show that $x \notin \mathbb{B}_{+}(E)$, more precisely, there exists $q \in \mathbb{N}_{>0}$ such that $\operatorname{Sym}^{q}(E) \otimes A^{-1}$ is globally generated at $x$. This proof is similar to the proof of Theorem 4.1.

We take a local coordinate $\left(U ; z_{1}, \cdots, z_{n}\right)$ near $x$. Let $\phi=\eta(n+1) \log |z-x|^{2}$, where $\eta$ is a cut-off function such that $\eta \equiv 1$ near $x$. Put $\psi:=\frac{n}{n+1} \pi^{*} \phi$. Let $h_{A}$ be a smooth positive Hermitian metric on $A$. We take $m \in \mathbb{N}_{>0}$ such that

(1) $m \sqrt{-1} \Theta_{A, h_{A}}+\sqrt{-1} \partial \bar{\partial} \eta \geq 0$ in the sense of current, and

(2) $\mathcal{O}_{\mathbb{P}(E)}(r) \otimes \pi^{*}\left(A^{-1} \otimes K_{X}^{-1} \otimes \operatorname{det} E^{*} \otimes A^{m}\right)$ has a smooth positive metric $h_{0}$.

We will denote by $g$ the singular Hermitian metric with semipositive curvature current on $O_{\mathbb{P}(E)}(k) \otimes A^{-1}$ induced by $h$. From $v_{\text {sup }}(h, x)=0$, there exists a open set $x \in V \subset \subset U$ such that $g^{2 m}$ is integrable on $\pi^{-1}(V)$ by Skoda's Theorem. Set

$$
\widetilde{L}:=\left(O_{\mathbb{P}(E)}(2 k m) \otimes \pi^{*} A^{-2 m}\right) \otimes\left(O_{\mathbb{P}(E)}(r) \otimes \pi^{*}\left(A^{-1} \otimes K_{X}^{-1} \otimes \operatorname{det} E^{*} \otimes A^{m}\right)\right) \otimes \pi^{*} A^{m}
$$

and $\widetilde{h}:=g^{2 m} h_{0} \pi^{*}\left(h_{A}^{m}\right)$. Then we have

$$
\begin{aligned}
\mathcal{O}_{\mathbb{P}(E)}(2 k m) \otimes \pi^{*} A^{-1} & =K_{\mathbb{P}(E)} \otimes \mathcal{O}_{\mathbb{P}(E)}(2 k m) \otimes \pi^{*} A^{-2 m} \otimes \mathcal{O}_{\mathbb{P}(E)}(r) \otimes \pi^{*}\left(A^{-1} \otimes K_{X}^{-1} \otimes \operatorname{det} E^{*} \otimes A^{m}\right) \otimes \pi^{*} A^{m} \\
& =K_{\mathbb{P}(E)} \otimes \widetilde{L}
\end{aligned}
$$

By the same argument of Theorem 4.1, $\widetilde{L}$ has a singular Hermitian metric $\widetilde{h}$ with semipositive curvature current such that

$$
\sqrt{-1} \Theta_{\widetilde{L}, \widetilde{h}}+\left(1+\frac{\alpha}{n}\right) \sqrt{-1} \partial \bar{\partial} \psi \geq 0 \text { in the sense of current }
$$


for any $\alpha \in[0,1]$. In this setting, $\operatorname{Sym}^{2 k m}(E) \otimes A^{-1}$ is globally generated at $x$, since the same proof as in Step 3 of $(\mathrm{C}) \Rightarrow(\mathrm{A})$ in Theorem 4.1 works.

It would be interesting to be able to describe non nef locus by using singular Hermitian metrics as in Theorem 6.8 .

\section{REFERENCES}

[BKK+15] T. Bauer, S. J Kovács, A. Küronya, E. C. Mistretta, T. Szemberg, S. Urbinati. On positivity and base loci of vector bundles. Eur. J. Math. 1 (2015), no. 2, 229-249.

[Ber20] B. Berndtsson. Lelong numbers and vector bundles. J. Geom. Anal. 30 (2020), no. 3, 2361-2376.

[Bou02] S. Boucksom. Cônes positifs des variétés complexes compactes. Thesis, Grenoble. (2002).

[Bou04] S. Boucksom. Divisorial Zariski decompositions on compact complex manifolds. Ann. Sci. École Norm. Sup. (4) 37 (2004), no. 1, 45-76.

[BDPP13] S. Boucksom, J.-P. Demailly, M. Păun, T. Peternell. The pseudo-effective cone of a compact Kähler manifold and varieties of negative Kodaira dimension. J. Algebraic Geom. 22 (2013), no. 2, 201-248.

[CDM17] J.-Y. Cao, J.-P. Demailly, S. Matsumura. A general extension theorem for cohomology classes on non reduced analytic subspaces. Sci. China Math. 60 (2017), no. 6, 949-962.

[CMSB02] K. Cho, Y. Miyaoka, N. I. Shepherd-Barron. Characterizations of projective space and applications to complex symplectic manifolds, Higher dimensional birational geometry. Adv. Stud. Pure Math. Math. Soc. Japan. 35 (2002), 1-88.

[CP17] J. Cao, M. Păun. Kodaira dimension of algebraic fiber spaces over abelian varieties. Invent. Math. 207 (2017), no. 1, 345-387.

[Dem82] J.-P. Demailly. Estimations $L^{2}$ pour l'opérateur $\bar{\partial}$ d'un fibré vectoriel holomorphe semi-positif au-dessus d'une variété kählérienne compléte. Ann. Sci. École Norm. Sup. (4) 15 (1982), no. 3, 457-511.

[Dem92] J.-P. Demailly. Singular Hermitian metrics on positive line bundles. Lecture Notes in Math. 1507 (1992), Springer, Berlin. 87-104.

[Dem12] J.-P. Demailly. Analytic methods in algebraic geometry. Surveys of Modern Mathematics. 1 (2012), viii+231.

[Dembook] J.-P. Demailly. Complex analytic and differential geometry. https://www-fourier.ujf-grenoble.fr/ demailly/manuscripts/agbook.pdf

[DPS94] J.-P. Demailly, T. Peternell, M. Schneider. Compact complex manifolds with numerically effective tangent bundles. J. Algebraic Geom. 3 (1994), no. 2, 295-345.

[DPS01] J.-P. Demailly, T. Peternell, M. Schneider. Pseudo-effective line bundles on compact Kähler manifolds. Internat. J. Math. 12 (2001), no. 6, 689-741.

[FM21] M. Fulger, T. Murayama. Seshadri constants for vector bundles. J. Pure Appl. Algebra 225 (2021), no. 4, $106559,35 \mathrm{pp}$.

[Gri69] P. A. Griffiths. Hermitian differential geometry, Chern classes, and positive vector bundles. 1969 Global Analysis (Papers in Honor of K. Kodaira) pp. 185-251 Univ. Tokyo Press, Tokyo

[HPS18] C. Hacon, M. Popa, C. Schnell. Algebraic fiber spaces over abelian varieties: around a recent theorem by Cao and Păun. Local and global methods in algebraic geometry, 143-195, Contemp. Math., 712, Amer. Math. Soc., Providence, RI, 2018.

[HIM19] G. Hosono, M. Iwai, S. Matsumura. On projective manifolds with pseudo-effective tangent bundle. Preprint, arXiv:1908.06421 to appear in Journal of the Institute of Mathematics of Jussieu.

[Hos17] G. Hosono. Approximations and examples of singular Hermitian metrics on vector bundles. Ark. Mat. 55 (2017), no. 1, 131-153.

[Iwa20] M. Iwai. On the global generation of direct images of pluri-adjoint line bundles Mathematische Zeitschrift. 294 201-208 (2020)

[Kod54] K. Kodaira. On Kähler varieties of restricted type (an intrinsic characterization of algebraic varieties). Ann. of Math. (2) 60 (1954), 28-48.

[Laz04a] R. Lazarsfeld. Positivity in algebraic geometry. I. Classical setting: line bundles and linear series. Ergebnisse der Mathematik und ihrer Grenzgebiete. 3. Folge. A Series of Modern Surveys in Mathematics [Results in Mathematics and Related Areas. 3rd Series. A Series of Modern Surveys in Mathematics], 48. Springer-Verlag, Berlin, (2004), xviii+387. 
[Laz04b] R. Lazarsfeld. Positivity in algebraic geometry. II. Positivity for Vector Bundles, and Multiplier Ideals. Ergebnisse der Mathematik und ihrer Grenzgebiete. 3. Folge. A Series of Modern Surveys in Mathematics [Results in Mathematics and Related Areas. 3rd Series. A Series of Modern Surveys in Mathematics], 48. Springer-Verlag, Berlin, (2004), xviii+387.

[LSY13] K. Liu, X. Sun, X. Yang. Positivity and vanishing theorems for ample vector bundles. J. Algebraic Geom. 22 (2013), no. 2, 303-331.

[Nak04] N. Nakayama. Zariski-decomposition and abundance. MSJ Memoirs, 14 (2004), Mathematical Society of Japan, Tokyo.xiv+277.

[PT18] M. Păun, S. Takayama, Positivity of twisted relative pluricanonical divisors and their direct images, J. Algebraic Geom. 27 (2018), 211-272.

[Vie83a] E. Viehweg. Weak positivity and the additivity of the Kodaira dimension for certain fibre spaces. Adv. Stud. Pure Math., 1, (1983), North-Holland, Amsterdam. 329-353.

[Vie83b] E. Viehweg. Weak positivity and the additivity of the Kodaira dimension. II. The local Torelli map. Progr. Math., 39, (1983), Birkhäuser Boston, Boston, MA. 567-589.

Graduate School of Mathematical Sciences, The University of Tokyo, 3-8-1 Komaba, Tokyo, 153-8914, Japan.

Email address: masataka@ms.u-tokyo.ac.jp, masataka.math@gmail.com 